\title{
A Novel 2D BEM with Composed Elements to Study Sloshing Phenomenon
}

\author{
M. Abbaspour ${ }^{1}$ and M. G. Hassanabad ${ }^{2}$ \\ School of mechanical engineering, Sharif University of Technology, Azadi Ave., Tehran, Iran. \\ Email: ${ }^{1}$ m-abbaspour@jamejam.net, ${ }^{2}$ ghodsi@mech.sharif.edu
}

(Received November 5, 2008; accepted January 9, 2009)

\begin{abstract}
In the Boundary Element Method (BEM), the corner elements make some difficulties in the numerical method. It means that, the results of BEM solution with linear elements are more accurate than constant elements inherently, but the difference between boundary conditions of one corner node from two adjacent boundaries causes to increase errors in linear element in comparison of constant element. In this article, a new approach of BEM discretizing has been introduced for simulating water sloshing in a 2D rectangular container which augments the accuracy. The time history of free surface shape has been obtained by applying Finite Difference Method (FDM) for free surface boundary conditions and BEM direct method with constant, linear and a novel element which has been named composed element in this study. Composed elements have been proposed for solving corner nodes problem. It means that all of the elements are linear except common nodes in two adjacent boundaries which are selected constant. This solution causes to decrease the number of elements for an acceptable precision and hence the time considerably saves potential flow and small amplitude water wave assumptions were applied in the present research. The results of this analysis illustrated acceptable agreement with other articles results with large dimension and small amplitude water wave assumptions. As a final point, the accuracy of results increased respect to linear, constant and composed elements.
\end{abstract}

Keywords: BEM, constant element, linear element, composed element, FDM.

\section{NOMENCLATURE}

$\begin{array}{ll}\Phi & \text { velocity potential function } \\ \xi & \text { amplitude of free surface } \\ \xi_{0} & \text { amplitude of the middle of free surface at } \\ & \text { initial time } \\ \mathrm{n} & \text { normal and outward direction to the boundary } \\ \mathrm{t} & \text { time }\end{array}$

\section{INTRODUCTION}

One of the most important phenomena in fluid mechanics is sloshing. The analysis of sloshing phenomena is important for the design of some fluid structure systems. For instance, the effects of this phenomenon in the LNG (Liquid Natural Gas) tanks of marine carriers are very important. The transportation of LNG in moving careers such as ships and vehicles has some technological problems. Graczyk and Moan (2008) have investigated the sloshing in LNG tanks and have concluded that the lower filled tanks create larger wave amplitude. The inherent motion of careers causes fluctuant forces in partially filled tanks as a result of sloshing. Therefore, one of the most important design parameters is the characteristics of liquid sloshing in a tank. The importance of the sloshing investigation is obvious in many other positions such as floating structures with liquid g gravitational acceleration

$\mathrm{k}$ iteration number

$\Psi \quad$ fundamental solution of Laplace equation

$\Omega \quad$ solution domain

$\Gamma \quad$ solution boundary

$\delta \quad$ Dirac delta tanks in the sea, dams during earthquake or after explosion, open end storage tanks in the sea bed, etc.

$\mathrm{BEM}$ is an appropriate computational method for solving sloshing as a subset of free surface flow, due to its lower solution time and well precision. Till now, BEM has been used for free surface flow analysis in many researches. Jamali (2006) has used FDM addition to BEM to model the motion of water free surface in a $2 \mathrm{D}$ rectangular container. Drevard et al. (2005) have analyzed the wave motion and wave breaking as a free surface flow phenomenon out of BEM. Hamano et al. (2003) have investigated the sloshing by BEM. Gedikli et al. (2003) have applied variational BEM to investigate the sloshing phenomena. Kukner and Baycal (1999) have showed that a triangular bed in a $2 \mathrm{D}$ rectangular tank can help to regulate the flow in tank by BEM. 
By considering the previous research works, in addition to other methods, new methods can be derived for removing BEM solution difficulties such as singularity and corner defects. The joint nodes in the adjacent boundaries (corner nodes) have two boundary values from two adjacent boundaries. If a relevant method should be used for dealing with this paradox, the related errors would be prevented in numerical method.

In the present study, a novel method has been proposed to tackle the corner difficulties. In this method, all elements have been assumed linear except corner elements which have been assumed constant. This new method of discretizing of boundaries has been named composed discretization and the elements have been named composed elements.

Using constant elements in corners removes the obstacle of bi-valued boundary conditions for one node, and using higher order elements for other elements, increases the precision of the numerical method.

\section{MATERIALS AND METHODS}

\subsection{Mathematical formulation}

Base on the potential theory fluid mechanics, the governing equation in sloshing problem is assumed Laplace equation. As mentioned in related references, the BEM has been based on the Laplace equation (Katsikadelis 2002; Brebbia and Dominguez 1992). In this research, the potential equation has been used as governing equation as bellow:

$$
\nabla^{2} \Phi=0
$$

Figure 1 shows a rectangular container. In this $2 \mathrm{D}$ container, water free surface has a triangular shape at initial state which can be expressed as:

$$
\xi(x, 0)=\xi_{0}\left(\frac{1}{2}-\frac{|x|}{L}\right)
$$

In this container, the water free surface falls down with zero initial velocity. The boundary conditions are expressed as bellow.

The boundary condition on sides and bottom walls is:

$$
\frac{\partial \Phi}{\partial n}=0
$$

By assuming small amplitude water wave, the kinematic and dynamic boundary conditions are:

$$
\begin{aligned}
& \frac{\partial \xi}{\partial t}=\frac{\partial \Phi}{\partial n}=\frac{\partial \Phi}{\partial z} \\
& \frac{\partial \Phi}{\partial t}=-g \xi
\end{aligned}
$$

According to finite difference relations and Eq. 4, the Eq. 5 is obtained as bellow:

$$
\begin{gathered}
\Phi^{k+1}=\Phi^{k}-g \Delta t \xi^{k}-\frac{g(\Delta t)^{2}}{2} \times \\
{\left[a\left(\frac{\partial \Phi}{\partial n}\right)^{k+1}+b\left(\frac{\partial \Phi}{\partial n}\right)^{k}\right]+O(\Delta t)^{3}}
\end{gathered}
$$

According to Salmon et al. (1980) research, the optimum values of "a" and "b" for stability and accuracy in the numerical method are 0.17 and 0.83 , respectively.

\subsection{Boundary element method}

In this study, BEM is based on Laplace equation and second Green's identity which can be expressed as follows:

$$
\begin{aligned}
& \int_{\Omega}\left(\Psi \nabla^{2} \Phi-\Phi \nabla^{2} \Psi\right) d \Omega= \\
& \oint_{\Gamma}\left(\Psi \frac{\partial \Phi}{\partial n}-\Phi \frac{\partial \Psi}{\partial n}\right) d s
\end{aligned}
$$

$\Psi$ is obtained from bellow.

$$
\nabla^{2} \Psi=\delta(q-p)
$$

In which "p" is source point. By considering Eqs. 6 and 7, the integral equation of BEM is obtained as bellow:

$$
\begin{aligned}
& \frac{\alpha}{2 \pi} \Phi(p)=-\int_{\Gamma}\left[\Psi(p, q) \frac{\partial \Phi(q)}{\partial n_{q}}-\right. \\
& \left.\Phi(q) \frac{\partial \Psi(p, q)}{\partial n_{q}}\right] d s_{q}
\end{aligned}
$$

In which $\Phi(p)$ is potential function in point "p" should be determined. "q" is an arbitrary node on the boundary and $\alpha$ is the angle with vertex at point "p" that sweeps the solution domain. If "p" is in the solution domain, on the smooth boundary or out of the domain, the $\alpha$ will be $2 \pi, \pi$ or 0 , respectively (Katsikadelis 2002).

Eq. 8 can be discretized in different ways. The two main boundary elements used in BEM are constant and linear. These two types of elements are shown in Fig. 2 schematically. Element geometry and $\Phi$ variation along the element are two important parameters in BEM. In each element, the positions of nodal points and extreme points determine all properties of element. Free and $\Phi_{n}$ (normal derivative of $\Phi$ ) in each element are assigned to nodal points. A constant element is a finite line which has a nodal point in the middle. Also the values of $\Phi$ and $\Phi_{n}$ are constant on the element. In the linear element, there are two nodal points on the extreme points of the element which $\Phi$ and $\Phi_{\mathrm{n}}$ vary linearly on it.

The discretized form of integral equation (Eq. 8) for constant element is: 


$$
\begin{aligned}
& -\frac{1}{2} \Phi^{i}+\sum_{j=1}^{N}\left(\int_{\Gamma_{j}} \frac{\partial \Psi\left(p_{i}, q\right)}{\partial n_{q}} d s_{q}\right) \Phi^{j}= \\
& \sum_{j=1}^{N}\left(\int_{\Gamma_{j}} \Psi\left(p_{i}, q\right) d s_{q}\right) \Phi_{n}^{j}
\end{aligned}
$$

The discretized form of integral equation (Eq. 8) for linear element is:

$$
\begin{aligned}
& -\frac{1}{2} \Phi^{i}+\sum_{j=1}^{N}\left[\left(\int_{\Gamma_{j}} \frac{\partial \Psi\left(p_{i}, q\right)}{\partial n_{q}} G_{1} d s_{q}\right) \Phi^{j}\right. \\
& \left.+\left(\int_{\Gamma_{j}} \frac{\partial \Psi\left(p_{i}, q\right)}{\partial n_{q}} G_{2} d s_{q}\right) \Phi^{j+1}\right] \\
& =\sum_{j=1}^{N}\left[\left(\int_{\Gamma_{j}} \Psi\left(p_{i}, q\right) G_{1} d s_{q}\right) \Phi_{n}^{j}\right. \\
& \left.+\left(\int_{\Gamma_{j}} \Psi\left(p_{i}, q\right) G_{2} d s_{q}\right) \Phi_{n}^{j+1}\right]
\end{aligned}
$$

In which $\mathrm{G}_{1}$ and $\mathrm{G}_{2}$ are:

$$
\begin{aligned}
& G_{1}=\frac{1-\xi}{2} \quad ; G_{2}=\frac{1+\xi}{2} \\
& \Psi=\frac{\ln |r|}{2 \pi} \quad ; \frac{\partial \Psi}{\partial n}=\frac{\cos \theta}{2 \pi|r|}
\end{aligned}
$$

In which " $r$ " is displacement vector from node $\mathrm{i}$ to $\mathrm{j}^{\text {th }}$ element and $\theta$ is the angle between " $n$ " and " $r$ ".

The composed element uses both of Eqs. 9 and 10 .

\subsection{Applying the composed element method}

The mathematical Eq. 9 is converted to a set of algebraic linear equation which can be solved by Gauss elimination method. In this set of equations, there is an unknown boundary value (depending on Dirichlet or Neumann boundary values) or a relation between Dirichlet and Neumann boundary values (Robin boundary condition) in each of its rows. The Eq. 9 can be shown in a matrix form as (Katsikadelis 2002):

$$
\left[H_{\text {Cons tan } t}\right]\{\Phi\}=\left[R_{\text {Cons tan } t}\right]\left\{\Phi_{n}\right\}
$$

In which:

$$
\begin{aligned}
& H_{\text {Cons tant }}^{i j}=-\frac{1}{2} \delta_{i j}+\int_{\Gamma_{j}} \frac{\partial \Psi\left(p_{i}, q\right)}{\partial n_{q}} d s_{q} \\
& R_{\text {Cons tant }}^{i j}=\int_{\Gamma_{j}} \Psi\left(p_{i}, q\right) d s_{q}
\end{aligned}
$$

Similar to Eq. 9, the mathematical Eq. 10 is converted to a set of algebraic linear equations as:

$$
\left[H_{\text {Linear }}\right]\{\Phi\}=\left[R_{\text {Linear }}\right]\left\{\Phi_{n}\right\}
$$

In which:

$$
\begin{aligned}
& H_{\text {Linear }}^{i j}=-\frac{1}{2} \delta_{i j}+ \begin{cases}h_{1}^{i 1}+h_{2}^{i N} & ; j=1 \\
h_{1}^{i j}+h_{2}^{i, j-1} & ; j=2 \text { to } N\end{cases} \\
& R_{\text {Linear }}^{i j}= \begin{cases}r_{1}^{i 1}+r_{2}^{i N} ; j=1 \\
r_{1}^{i j}+r_{2}^{i, j-1} & ; j=2 \text { to } N\end{cases}
\end{aligned}
$$

In which $\mathrm{N}$ is the number of elements and:

$$
\begin{aligned}
& h_{1}^{i j}=\int_{\Gamma_{j}} \frac{\partial \Psi\left(p_{i}, q\right)}{\partial n_{q}} G_{1} d s_{q} \\
& h_{2}^{i j}=\int_{\Gamma_{j}} \frac{\partial \Psi\left(p_{i}, q\right)}{\partial n_{q}} G_{2} d s_{q} \\
& r_{1}^{i j}=\int_{\Gamma_{j}} \Psi\left(p_{i}, q\right) G_{1} d s_{q} \\
& r_{2}^{i j}=\int_{\Gamma_{j}} \Psi\left(p_{i}, q\right) G_{2} d s_{q}
\end{aligned}
$$

These two methods are common in BEM. The second method is more accurate than the first one but has the corner node problem. There are various solutions for this problem, two instances of which are using double nodes on one corner and using discontinuous elements on corners (Hamano 2003 and Lefeber 1989).

The idea of this article is a new solution. In this approach, it is not necessary to define a new format for elements. It can use traditional constant elements and linear elements, as shown in Fig. 3. This method is simpler than other methods. In this figure, the corner elements are constant and the others are linear. For this purpose, it is necessary to compose $\mathrm{H}_{\text {Constant }}$ and $\mathrm{H}_{\text {Linear }}$ matrices and $\mathrm{R}_{\text {Constant }}$ and $\mathrm{R}_{\text {Linear }}$ matrices as mentioned in Eqs. 12 and 14 .

Therefore, in each boundary such as free surface, the initial and end elements are constant and the others are linear. In the present study, the composition form has been derived for each boundary as bellow:

$$
\begin{aligned}
& H_{\text {Composed }}^{i j}=H_{\text {Cons tan } t}^{i 1}+\left(-\frac{1}{2} \delta_{i 2}+h_{1}^{i 2}\right) \\
& +H_{\text {Linear }}^{i j}+\left(-\frac{1}{2} \delta_{i N_{b}}+h_{1}^{i N_{b}}\right)+H_{\text {Cons tan } t}^{i, N_{b}+1} \\
& R_{\text {Composed }}^{i j}=R_{\text {Cons tan } t}^{i 1}+r_{1}^{i 2}+R_{\text {Linear }}^{i j} \\
& +r_{1}^{i N_{b}}+R_{\text {Cons tan } t}^{i, N_{b}+1}
\end{aligned}
$$

in which $\mathrm{N}_{\mathrm{b}}$ is the number of elements in each boundary. In the specific instance of this research, there are 4 boundaries that the Eq. 17 must be applied for each of them.

The set of equations was solved by Gauss elimination method and hence it was not necessary to use any iterative method. In each time, the potential function of free surface was obtained. The velocity of free surface nodes was obtained from potential function and the displacement of them was calculated by time interval.

The "Spline" approximation was used to generate new free surface in each time step. 


\subsection{Research procedure}

In this study, the maximum amplitude of free surface $\left(\xi_{0}\right)$ as mentioned in Fig. 1 and Eq. 2 is 0.01. Also the linear boundary conditions have been used for free surface. The large dimensions of the container $(\mathrm{L}=9.8, \mathrm{H}=9.8)$ have been used so that the linear assumption of free surface would be valid.

The procedure of this article has 3 steps as bellow:

(1) The problem was solved in this study was investigated by constant element and compared to results obtained by Liu and Liggett (1982) as shown in Fig. 4.

(2) Linear element was used for analysis the problem of the present research and the results were compared with the results obtained by Liu and Liggett (1982) as shown in Fig. 5.

(3) The composed element (using combined constant and linear element) was used to increase the accuracy. According to Fig. 6 there is an excellent agreement between the results of present study and the results obtained by Liu and Liggett (1982).

The errors between Salmon et al. research and the present study were obtained from Eq. 18 for the results of three states mentioned above.

error $=\frac{\sqrt{\sum_{i=1}^{n}\left(\frac{z_{\text {numerical }}^{i}-z_{\text {analytical }}^{i}}{z_{\text {analytical }}^{i}}\right)^{2}}}{n}$

\section{RESULTS}

With respect to the firs step, the accuracy of the results was increased by increasing the free surface elements.

By following the second step and using Eq. 18, it is observed that the precision of constant element was better than linear element with the same number of elements, as shown in table 1.

Using third step along with Eq. 18 resulted in higher precision of composed element with respect to constant and linear elements with the same number of free surface elements, as shown in table 1 .

Figures 4 to 6 demonstrate the end nodes displacement of water free surface in the state of constant, linear and composed element in 10 seconds, respectively. In these figures, the results of the present study and the results of analytical solution (Liu and Liggett 1982) versus time have been drawn in each graph.

Table 1 and Figs. 4 to 6 indicate the improvement of results by using composed element. Also, Fig. 7 shows the free surface oscillation in sloshing problem of this article with 50 composed elements on free surface.

\section{DISCUTION AND CONCLUTION}

In the problem of the present research, divergence occurred when $\Delta \mathrm{t}$ was greater than $0.87 \mathrm{~s}$. The error values have been illustrated in table 2. Also, Fig. 8 shows the error according to Eq. 18, in term of $\log (\Delta t)$ by applying composed elements with 50 nodes on the free surface boundary.

In BEM, corner nodes difficulty can affect the results accuracies. The unknown potential function and its derivative are assigned to extreme nodes in linear element. However, they are assigned to middle element in constant element. Therefore, two different boundary values are imposed on each corner nodes in linear elements. For instance, in this study, both kinematic and dynamic boundary conditions from free surface and walls boundary condition from side walls are imposed on the end points of free surface. This conflict leads to error in results. However, according Fig. 2, unknown values are related to middle node in constant element (there isn't any node in the corners) and hence, each node on the boundaries has a single boundary value.

Hamano et al. (2003) have used two methods for corner nodes problem in BEM. The first method is to use double nodes and the second method is to use discontinuous element. They showed that using discontinuous elements is more stable than using double nodes. Also, the second method is not always successful in giving reliable solution according to some researches (Lefeber 1989). The constant element usually solves all types of BEM problems but it is the most inaccurate among other BEM methods due to elements discontinuity.

This study, the composed element idea was propounded to improving the accuracy of the solution. In this new method, discontinuity of boundary values in corner elements which is the nature of constant elements can be occurred. But the results of the present research show that the accuracy of these types of elements was significantly improved compared to linear and constant elements.

\section{REFERENCES}

Brebbia, C.A., J. Dominguez (1992). Boundary Elements: An Introductory Course. Southampton: Computational Mechanics Publications, McGrawHill.

Drevard, D., R. Marcer, S.T. Grilli, M. Asce, P. Fraunie, and V. Rey (2005). Experimental validation of a coupled BEM-Navier-stokes model for solitary wave sloshing and breaking. Ocean Waves Measurement and Analysis, 5th Int. Symposium Waves, 166-175.

Gedikli, A., M.E. Erguven (2003). Evaluation of sloshing problem by variational boundary element method. Engineering Analysis with Boundary Elements 27, 935-943.

Graczyk, M. and T. Moan (2008). A probabilistic assessment of design sloshing pressure time histories in LNG tanks. Ocean Engineering 35, 834-855.

Hamano, K., S. Murashige, K. Hayami (2003). Boundary element simulation of large amplitude standing waves in vessels. Engineering Analysis with Boundary Elements 27, 565-574.

Jamali, M. (2006). BEM modeling of surface water wave motion with laminar boundary layers. Engineering Analysis with Boundary Elements 30, 14-21. 
M. Abbaspour and M.G. Hassanabad / JAFM, Vol. 2, No. 2, pp. 77-83, 2009.

Katsikadelis, J.T. (2002). Boundary Elements: Theory and Applications, Elsevier, First edition.

Kukner, A. and M.A. Baykal (1999). Wave sloshing in corrugated bottom tanks with two-dimensional flow. Ocean Engineering 26, 703-712.

Lefeber, D. (1989). Solving problems with singularities using boundary elements. In: Brebbia C.A. and J.J. Connor, Topics in engineering 6, Computational Mechanics Publications, Southampton.
Liu, P.L. and J.A. Liggett (1982). Application of boundary element methods to problems of water waves. Chapter 3 of: Banerjee P.K. and R.P. Shaw, Developments in boundary element methods-2, Applied science publishers LTD.

Salmon, J.R., P. Liu, and J.A. Liggett (1980). Integral equation method for linear water waves. $J$. Hydraulics Div, ASCE, 106, 1995-2010.

Table 1 Amounts of error (Eq. 18) for different element types and numbers.

\begin{tabular}{|c|c|c|}
\hline Element type & Number of free surface elements & Error \\
\hline \multirow{3}{*}{ Constant element } & 20 & 0.1633 \\
\cline { 2 - 3 } & 50 & 0.0285 \\
\cline { 2 - 3 } & 100 & 0.0208 \\
\hline \multirow{2}{*}{ Linear element } & 20 & 0.4640 \\
\cline { 2 - 3 } & 50 & 0.1158 \\
\hline \multirow{2}{*}{ Composed element } & 20 & 0.0924 \\
\cline { 2 - 3 } & 50 & 0.0098 \\
\hline
\end{tabular}

Table 2 Amounts of error (Eq. 18) for different time steps.

\begin{tabular}{|c|c|c|c|c|c|c|c|c|}
\hline$\Delta$ t (sec) & 0.0001 & 0.0005 & 0.001 & 0.005 & 0.01 & 0.05 & 0.1 & 0.2 \\
\hline Error & 0.0082 & 0.0083 & 0.0084 & 0.0086 & 0.0089 & 0.0091 & 0.0095 & .0098 \\
\hline$\Delta$ t (sec) & 0.3 & 0.4 & 0.5 & 0.6 & 0.7 & 0.8 & 0.87 & \multirow{2}{*}{ Diverged } \\
\hline Error & 0.0112 & 0.039 & 0.097 & 0.208 & 0.411 & 0.752 & 0.954 & \\
\hline
\end{tabular}

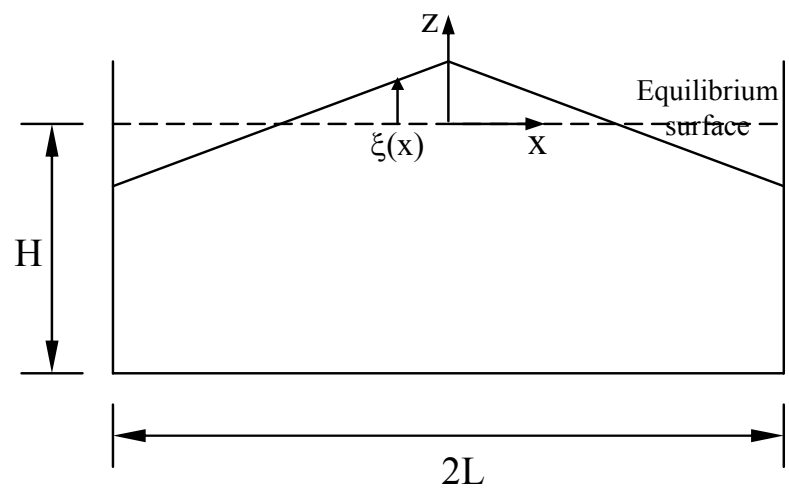

Fig. 1. Initial shape of free surface in rectangular container

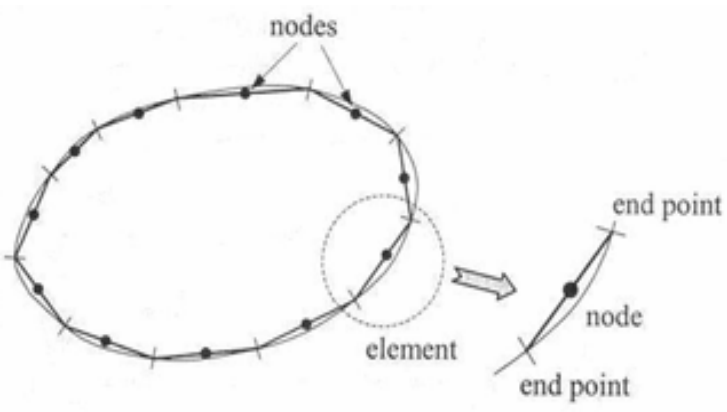

Constant element

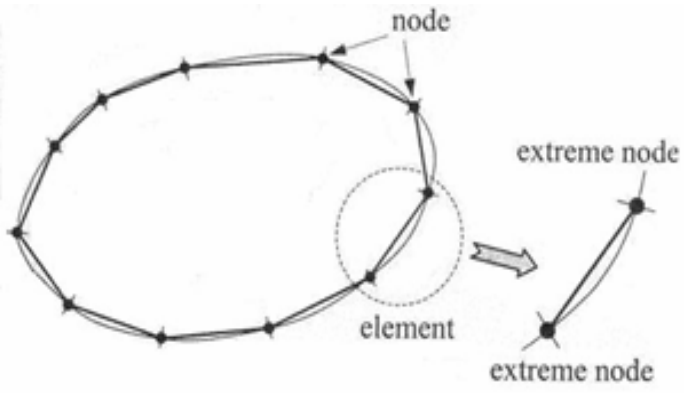

Linear element

Fig. 2. Schematic shape of BEM constant and linear elements (Katsikadelis 2002). 
M. Abbaspour and M.G. Hassanabad / JAFM, Vol. 2, No. 2, pp. 77-83, 2009.

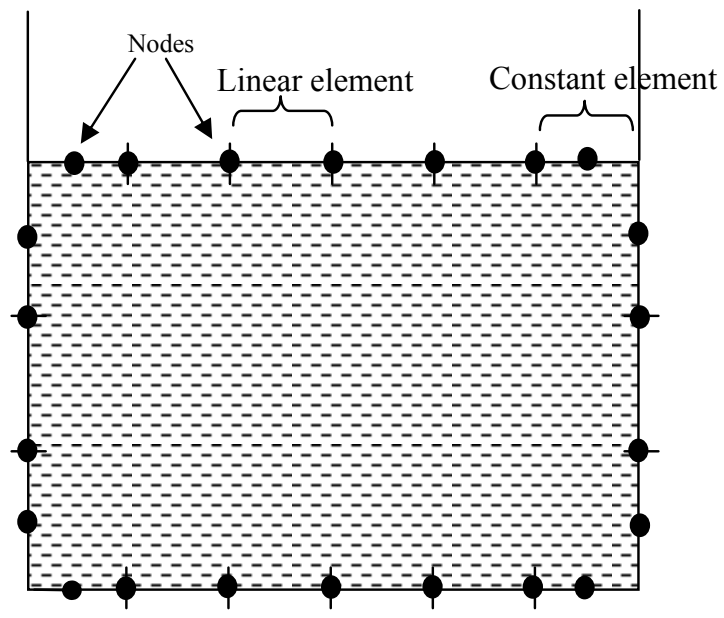

Fig. 3. The arrangement of nodes on the boundaries in composed format.

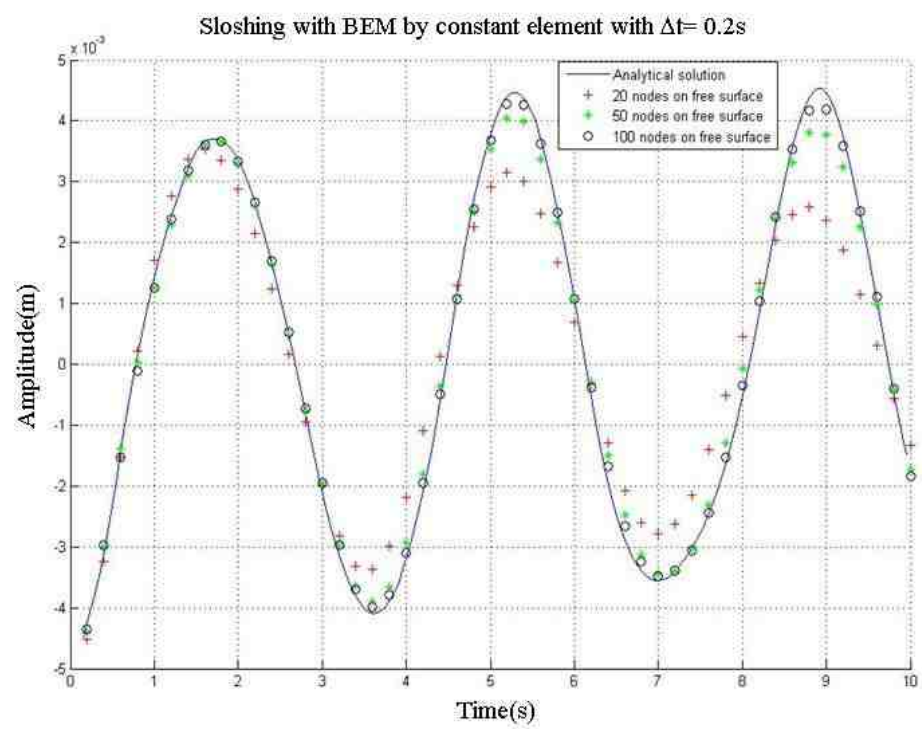

Fig. 4. Free surface end points displacement $(x=L, x=-L)$ during $10 \mathrm{~s}$ with constant element.

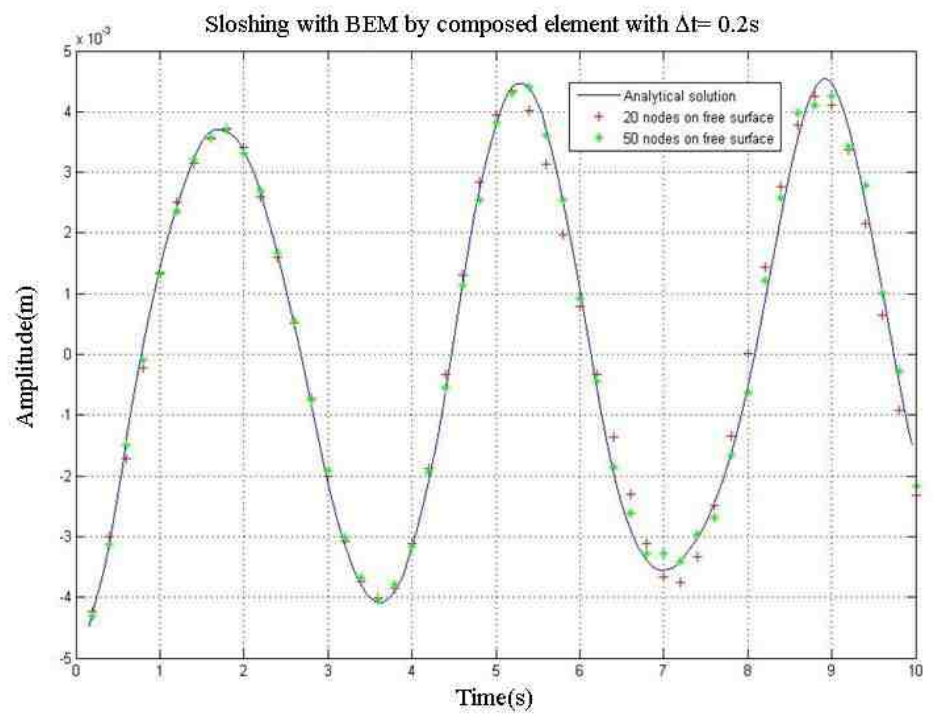

Fig. 5. Free surface end points displacement $(x=L, x=-L)$ during 10 s with linear element. 
M. Abbaspour and M.G. Hassanabad / JAFM, Vol. 2, No. 2, pp. 77-83, 2009.

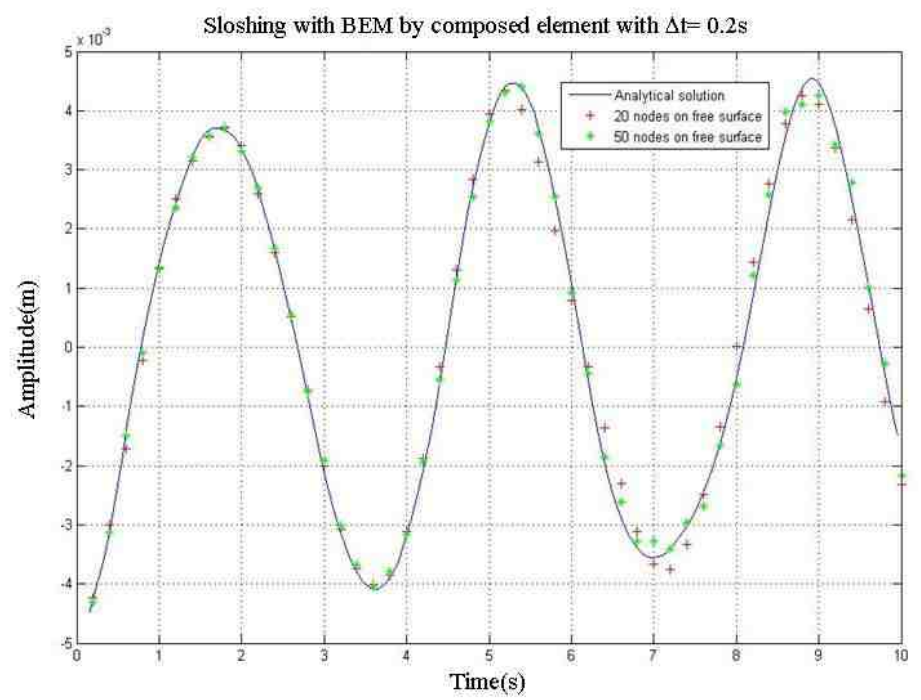

Fig. 6. Free surface end points displacement $(x=L, x=-L)$ during $10 \mathrm{~s}$ with composed element.

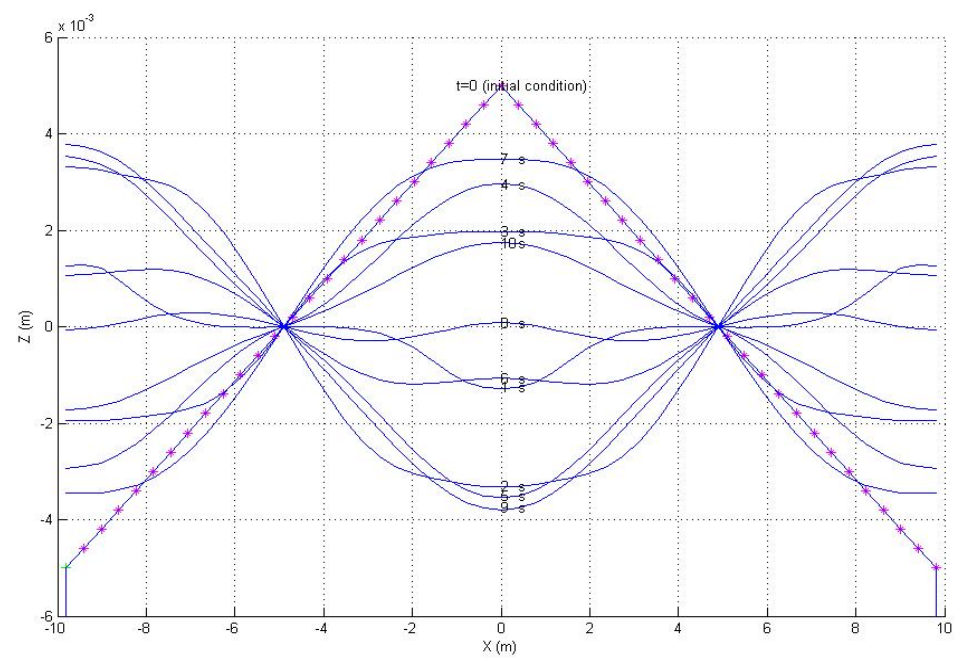

Fig. 7. The oscillation of free surface in sloshing phenomena with 50 elements on it.

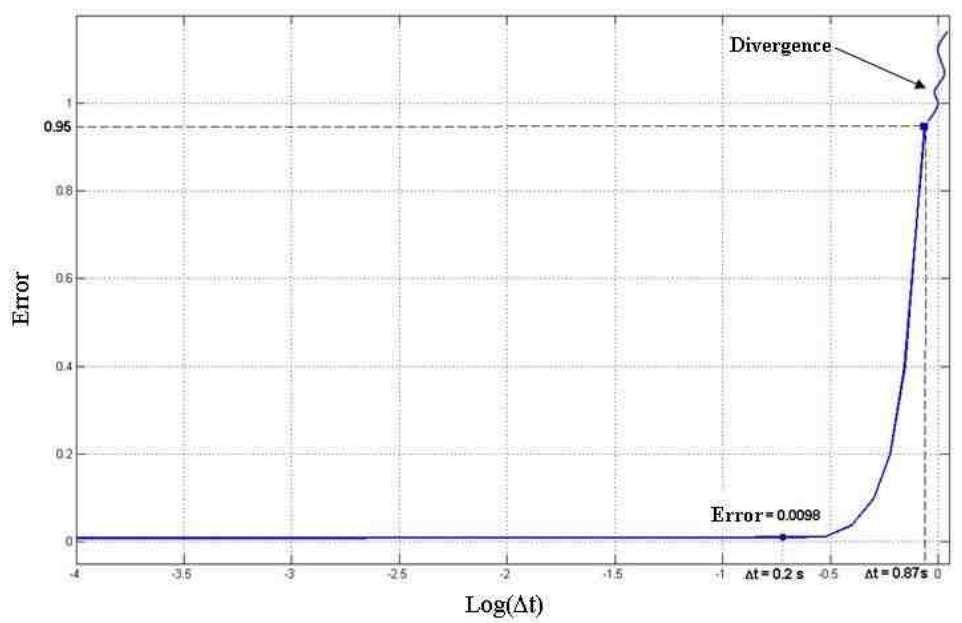

Fig. 8. Error of solution (Eq. 18) versus time step logarithm. 